\title{
Grumpy Old Cells
}

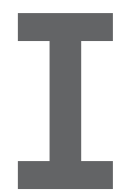

AM GoING way out on a limb in this column into an area where I really know very little but am completely fascinated by what I am learning. The tenuous linkage to our discipline is what I will call programmed cell self-destruction or maybe cell suicide.

I have been reading at length a book called Molecular Biology of the Cell. This is a 1,342-page book, not counting index and glossary and a separate book of problems. It is profusely illustrated and eminently readable even by a layperson like me, pretending, of course, that I am actually understanding what I am reading.

It turns out that cells reproduce (that is, divide: mitosis) but usually only a finite number of times. When they divide, their DNA is duplicated within the cell and separate copies are transported into each new daughter cell. Human DNA comes in 23 distinct chromosomes. Each chromosome is made up of a double helix of DNA. During cell mitosis, each strand of the double helix is duplicated by figuratively unzipping the double helix and replicating each strand. The replication takes place at multiple replication origin sites along the strand so this process operates in parallel. The nowduplicated chromosomes look like elongated "X"-shaped Gumby characters formed by adjacent DNA strands. As the mitotic process continues, the duplicated chromosomes are pulled apart by microtubules that attach to opposite sides of the paired chromosomes. As the process proceeds, eventually two new nuclei form with its copy of the original cell's DNA and the cell completes its division into two essentially identical cells.

At the ends of each strand of DNA is a repetitive sequence of DNA called a telomere. There are multiple telomeres at each end of the chromosome. One might think of them figuratively as handles needed to anchor the DNA during the unzipping and replication process. The telomeres themselves are not replicated in this process, so every cell division may lose one or more telomeres. If there are too few telomeres left, the replication process fails and initiates a process known as cell apoptosis, which we can think of as programmed cell death. Interestingly, certain kinds of cells known as embryonic stem (ES) cells found in bone marrow and in the gut contain an enzyme called telomerase that fabricates new telomeres so that ES cells can replicate indefinitely. Non-ES cells, which make up most cells in our bodies, count down to termination of replication. Cancer cells manage to avoid this outcome by using telomerase to make more telomeres allowing indefinite proliferation.

Cellular apoptosis can be triggered within the cell or by outside factors

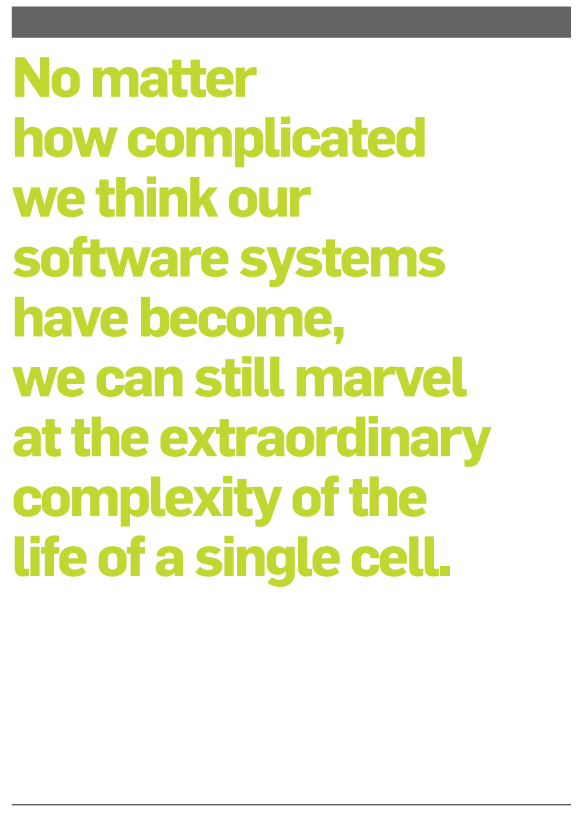

and the disintegrated debris, each enclosed in cell membranes, are consumed by phagocytes ("cell eaters"). It is estimated, for example, that phagocytes consume $10^{11}$ blood cells a day. Other cells experience necrosis, which is triggered from outside the cell causing the cell to rupture and spew its contents into intercellular space. One way this can happen is if cells have experienced some form of trauma. Viruses can invade cells, commandeer the DNA interpretation system (that is, ribosomes) with their own DNA, and eventually rupture the cell wall, broadcasting new virus particles into the surrounding tissues.

Once a cell has stopped replicating, it may not immediately experience either apoptosis or necrosis. Rather, it may continue to exist in a senescent state, which I have chosen to label an increasingly grumpy state. It may continue to produce proteins but they may prove to be harmful to other cells. The aging process and its manifest side effects can be traced, in part, to grumpy old cells spewing harmful products into the biological neighborhood. One thinks of the plaques and tangles of Alzheimer's disease and the misfolded prions associated with Creutzfeldt-Jakob encephalopathy caused by harmful proteins synthesized by grumpy cells.

If you got all the way to the end of this column, congratulations! No matter how complicated we think our software systems have become, we can still marvel at the extraordinary complexity of the life of a single cell and the immeasurable complexity of multicellular life, including our own.

Vinton G. Cerf is vice president and Chief Internet Evangelist at Google. He served as ACM president from 2012-2014. 